elective work, including TAVI services from March 2020. We sought to evaluate clinical outcomes and time delays in patients undergoing TAVI during the pandemic period compared to an age and risk factor-matched cohort of patients prior to COVID-19. We hypothesized that there were significant time delays, more emergency procedures and related adverse outcomes in patients who underwent TAVI during the pandemic period.

Methods We analysed prospectively collected data (patient characteristics, procedural details, complications and in-hospital outcomes) of 210 consecutive patients who underwent TAVI between March 2019 and February 2021 in a tertiary centre in the UK (The centre serves for a population of 2.5 million and provided in-patient treatment for 5590 COVID-positive patients over a 12-month period). We compared time-lags from an initial referral to outpatient review, CT aortograms, MDT discussions, valve implantations and 30-day mortality between patients who underwent TAVI between March 2019 and February 2020 (Control group=134) and those who underwent TAVI between March 2020 and February 2021 (COVID Group=76).

Results The mean age of the cohort was $81.9 \pm 6.4$ years compared to $80.9 \pm 6.9$ in the COVID-19 group. $59 \%$ of the control group and $43 \%$ of the COVID-19 group were female. Majority were in the moderate risk category (EuroSCORE $\mathrm{II}=4.55 \pm 5.5$ ). Mean Katz index in the control group was 5.4 \pm 0.9 and $5.7 \pm 0.5$ in the COVID-19 cohort. Patient characteristics are summarised in table 1 . Of the total cohort, $4(5.3 \%)$ patients acquired COVID-19 pneumonia during the hospital stay. The comorbidities and risk scores were comparable between the control group and the COVID cohort. (Table 1). There were no significant differences in procedural complications in the control group compared to the COVID-19 group (table 2). The median waiting time from referral to TAVI clinic was significantly shorter in the COVID-19 group (33 (8-66) vs. $51(17-89)$ days $(\mathrm{P}=0.04))$ and there was no significant difference in time delays for CT aortogram, MDT or

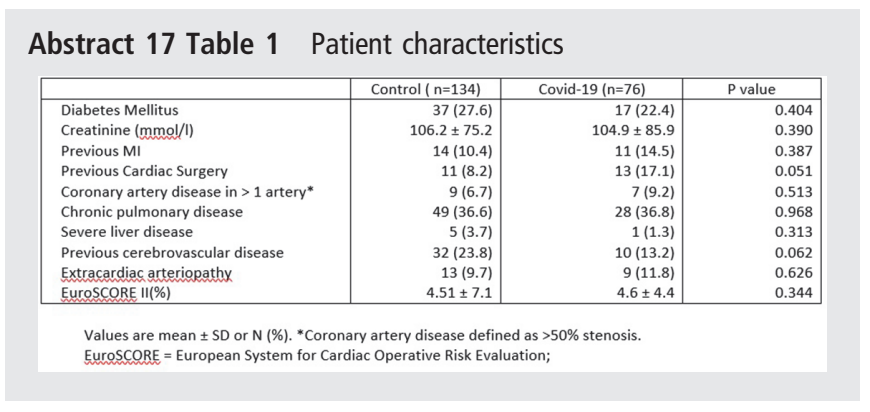

Abstract 17 Table 2 Complications and clinical outcomes

\begin{tabular}{|l|r|r|r|}
\hline Complications & Control $(\mathrm{n}=134)$ & Covid-19 (n=76) & P value \\
\hline Post procedural stroke & $6(4.5)$ & $2(2.6)$ & 0.439 \\
Major bleeding* & $3(2.2)$ & $2(2.6)$ & 0.926 \\
AKI Stage 2 and 3+ & $1(0.7)$ & $1(1.3)$ & 0.725 \\
Major vascular complications & $4(3)$ & $5(6.6)$ & 0.267 \\
Percutaneous device closure failure & $4(3)$ & $2(2.6)$ & 0.811 \\
New pacemaker implantation & $25(18.7)$ & $15(19.7)$ & 0.942 \\
Endocarditis & $1(0.7)$ & $0(0)$ & 0.433 \\
Cardiac tamponade & $0(0)$ & $1(1.3)$ & 0.2 \\
Valve malpositioning & $1(0.7)$ & $0(0)$ & 0.433 \\
\hline
\end{tabular}

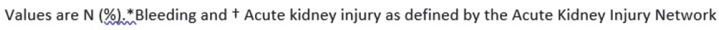
criteria. ₹ Major vascular complications as defined by the Valve Academic Research Consortium 2

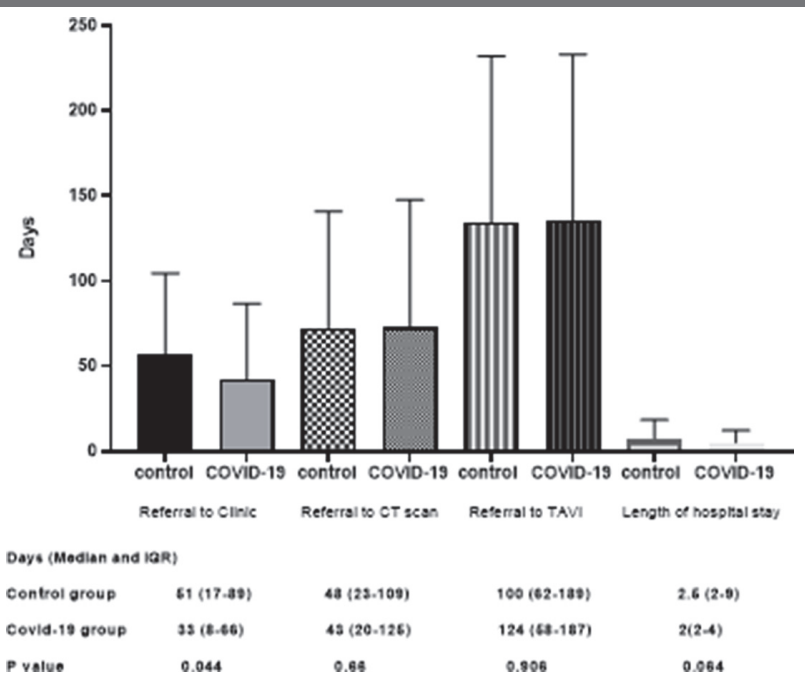

Abstract 17 Figure 1

TAVI procedure between the two groups (Figure 1). The median length of stay $(2(2-4)$ vs $2.5(2-9)$ days) and 30-day mortality $(1.4 \%$ vs $5.3 \%)$ was comparable between the two groups.

Conclusion Contrary to our hypothesis, our analysis demonstrated that there were no significant time delays, excess complications or mortality in TAVI procedures during the COVID-19 pandemic period despite the excess burden imposed on our local health services. More importantly, very few TAVI patients acquired COVID-19 sepsis during in-hospital stay. This is likely due to prompt identification of innovative ways of re-configuring an existing local patient pathway, by the TAVI team, to deliver safe and uninterrupted TAVI services during this unprecedented pandemic setting.

Conflict of Interest None

\section{PREVALENCE OF AORTIC ROOT DILATATION IN PATIENTS WITH FABRY DISEASE: A SINGLE CENTRE EXPERIENCE}

Christina Peter, Tian Zhao, Patrick Deegan, Rosemary Rusk. Cambridge University Hospitals, Cambridge, UK

\subsection{6/heartjnl-2021-BCS.18}

Introduction Fabry disease is an $\mathrm{X}$ linked lysosomal storage disorder resulting from deficiency of the enzyme alpha-galactosidase A. Multi-systemic effects are seen due to deposition and accumulation of glycosphingolipids. Although left ventricular hypertrophy is often the most commonly cited cardiac finding, cardiac manifestations can be widely varied, and vascular structural changes with increased thickening of the intima media and progressive vascular dilatation have been observed. Aortic root dilatation in affected males has in the past been reported in up to $30-56 \%$ using echocardiography, with varying criteria used to define dilatation.

Purpose To establish the prevalence of aortic root and ascending aortic dilatation evaluated by echocardiography in our cohort of Fabry patients.

Method We identified genotype positive Fabry patients from our single centre Fabry database who underwent 
echocardiography between January 2019 and January 2021. Data on basic demographics, history of hypertension, cardiac involvement and use of enzyme replacement therapy (ERT) were collected retrospectively. Cardiac involvement was defined as abnormalities detected on echocardiography, cardiovascular magnetic resonance imaging and /or elevated high sensitivity troponin I levels (reference limit $>40 \mathrm{ng} / \mathrm{L}$ for females and $>58 \mathrm{ng} / \mathrm{L}$ for males) not explained by another disease process. Imaging abnormalities included either left ventricular hypertrophy $\geq 13 \mathrm{~mm}$ and / or evidence of fibrosis. The maximal aortic root dimension was assessed at the level of the sinus of Valsalva (SOV) and the proximal ascending aorta using echocardiography. Dimensions $\geq 40 \mathrm{~mm}$, or with a $\mathrm{Z3}$ score $>2$ (normalised for height by Devereux et al), were classed as dilated. Dimensions are quoted as mean \pm standard deviation. Statistical analysis was through Chi-squared testing.

Results 82 patients were identified (31 male, 51 female) ages ranging between 23 and 83 years (mean of $51 \pm 16.1$ years). $21 \%$ had treated hypertension $(\mathrm{n}=17)$ and $62 \%$ had cardiac involvement from their Fabry disease $(\mathrm{n}=51)$. $54 \%$ were on ERT $(\mathrm{n}=44)$, of whom $70 \%(\mathrm{n}=31)$ were on intravenous enzyme replacement therapies and 30\% (n $=13$ ) were on oral chaperone therapy. The SOV dimensions ranged from 24 to $43 \mathrm{~mm}$ (mean $32 \pm 4.4 \mathrm{~mm}$ ). Aortic root dilatation was identified in $8.5 \%$ of the patient group $(\mathrm{n}=7)$. Ascending aortic dimensions beyond the root were available in 72 patients. 2 patients had ascending diameters $>40 \mathrm{~mm}$, but both also had aortic root dilatation. Table 1 outlines the prevalence of aortic root dilatation in our cohort grouped by gender, history of hypertension, cardiac involvement and use of ERT. The prevalence of aortic root dilatation in patients with cardiac involvement from their Fabry disease was significantly greater compared to patients with no cardiac involvement $(\mathrm{p}=0.03)$. The prevalence of aortic root dilatation was not statistically different when grouped by gender, history of hypertension or use of ERT.

\section{Abstract 18 Table 1}

\begin{tabular}{|l|c|}
\hline Subgroups & Dilated aortic root, $\mathbf{n}(\%)$ \\
\hline Male & $5(16.1)$ \\
Female & $2(3.9)$ \\
\hline History of hypertension & $1(5.9)$ \\
Nohistory of hypertension & $6(9.2)$ \\
\hline Cardiac involvement & $7(13.7)$ \\
Nocardiac involvement & $0(0)$ \\
\hline ERT & $6(13.0)$ \\
NoERT & $1(2.8)$ \\
\hline
\end{tabular}

Conclusion Fabry disease is a recognised lysosomal storage disorder associated with aortic root dilatation, although the exact mechanism remains incompletely understood. The prevalence of aortic root dilatation in our cohort was lower than previously reported. This may reflect advancements in treatment strategies and varying criteria used to define dilatation in previous studies. In our patient cohort the degree of aortic dilatation was mild, not reaching surgical requirement, and was not related to a history of hypertension. Although numbers were small, a higher prevalence was seen in patients with myocardial involvement by their Fabry disease, suggesting a possible link between the cardiac Fabry process and changes in the aortic wall.

Conflict of Interest none

\section{PROSPECTIVE LONGITUDINAL CHARACTERIZATION OF THE RELATIONSHIP BETWEEN DIABETES AND CARDIAC STRUCTURAL AND FUNCTIONAL CHANGES}

${ }^{1}$ Amrit Chowdhary, 'Nicholas Jex, 'Sharmaine Thirunavukarasu, 'Amanda MacCannell, ${ }^{1}$ Natalie haywood, ${ }^{1}$ Altaf Almutairi, ${ }^{2}$ Lavanya Atithan, 'Manali Jain, ${ }^{1}$ Thomas Craven, ${ }^{1}$ Arka Das, ${ }^{1}$ Noor Sharrack, ${ }^{1}$ Christopher Saunderson, ${ }^{3}$ Anshuman Sengupta, ${ }^{1}$ Lee Roberts, ${ }^{1}$ Peter Swoboda, ${ }^{4}$ Richard Cubbon, 'Klaus Witte, 'John Greenwood, 'Sven Plein, ${ }^{5}$ Eylem Levelt. ${ }^{1}$ University of Leeds, Leeds, UK; ${ }^{2}$ University of Leicester; ${ }^{3}$ Leeds Teaching Hospitals NHS Trust; ${ }^{4}$ Discovery and Translational Science Department Leeds Institute of Cardiovascular and Metabolic Medic; ${ }^{5}$ University of Leeds, Multidisciplinary Cardiovascular Research Centre and Biomedical Imaging Science

\subsection{6/heartjnl-2021-BCS.19}

Objectives In a cohort of type 2 diabetes (T2D) patients who underwent baseline cardiac magnetic resonance (CMR) and biomarker testing, during a median follow-up of 6-years we aimed to determine longitudinal changes in the phenotypic expression of heart disease in diabetes; report clinical outcomes; and compare baseline clinical characteristics and CMR findings of patients who experienced major adverse cardiovascular events (MACE) to those remaining MACE free (figure 1).

Background T2D increases the risk of heart failure (HF) and cardiovascular mortality. The long-term impact of T2D on cardiac phenotype in the absence of cardiovascular disease and other clinical events is unknown.

\begin{tabular}{|c|c|c|c|}
\hline Variable & $\begin{array}{l}\text { Baseline } \\
(\mathrm{n}=32)\end{array}$ & $\begin{array}{l}\text { Follow up } \\
(\mathrm{n}=32)\end{array}$ & $P$ value \\
\hline LV end diastolic volume (ml) & $159 \pm 29$ & $145 \pm 22$ & $0.005^{*}$ \\
\hline LV end diastolic volume index $\left(\mathrm{ml} / \mathrm{m}^{2}\right)$ & $78 \pm 12$ & $73 \pm 10$ & $0.02^{*}$ \\
\hline LV end systolic volume (ml) & $64 \pm 16$ & $65 \pm 19$ & 0.5 \\
\hline LV end systolic volume index $\left(\mathrm{ml} / \mathrm{m}^{2}\right)$ & $31 \pm 7$ & $33 \pm 9$ & 0.3 \\
\hline LV stroke volume $(\mathrm{ml})$ & $95 \pm 20$ & $80 \pm 14$ & $0.001^{*}$ \\
\hline $\begin{array}{l}\text { LV ejection fraction (\%) } \\
\Delta \text { LVEF (\%) }\end{array}$ & $60 \pm 7$ & $\begin{array}{l}55 \pm 8 \\
-(5.66 \pm \\
4.38)\end{array}$ & $0.0001^{*}$ \\
\hline LV mass (gm) & $102 \pm 17$ & $94 \pm 16$ & $0.01^{*}$ \\
\hline $\mathrm{LV}$ mass index $\left(\mathrm{gm} / \mathrm{m}^{2}\right)$ & $51 \pm 8$ & $47 \pm 8$ & $0.04^{*}$ \\
\hline $\begin{array}{l}\text { LV mass to LV end diastolic volume }(\mathrm{gm} / \\
\mathrm{ml} \text { ) }\end{array}$ & $0.65 \pm 0.12$ & $0.66 \pm 0.14$ & 0.8 \\
\hline $\begin{array}{l}\text { Peak diastolic circumferential strain rate } \\
(1 / s)\end{array}$ & $0.98 \pm 0.28$ & $1.04 \pm 0.23$ & 0.4 \\
\hline Peak diastolic longitudinal strain rate $(1 / s)$ & $0.75 \pm 0.21$ & $0.69 \pm 0.16$ & 0.2 \\
\hline RV end diastolic volume (ml) & $166 \pm 33$ & $142 \pm 25$ & $0.03^{*}$ \\
\hline RV end diastolic volume index $\left(\mathrm{ml} / \mathrm{m}^{2}\right)$ & $82 \pm 14$ & $71 \pm 12$ & $0.0001^{*}$ \\
\hline RV end systolic volume (ml) & $76 \pm 18$ & $70 \pm 16$ & $0.05^{*}$ \\
\hline $\mathrm{RV}$ end systolic volume index $\left(\mathrm{ml} / \mathrm{m}^{2}\right)$ & $37 \pm 8$ & $35 \pm 8$ & 0.1 \\
\hline RV stroke volume $(\mathrm{ml})$ & $91 \pm 20$ & $72 \pm 15$ & $<0.0001^{*}$ \\
\hline RV ejection fraction (\%) & $55 \pm 5$ & $51 \pm 7$ & $0.003^{*}$ \\
\hline$\triangle \operatorname{RVEF}(\%)$ & & $\begin{array}{l}-(6.69 \pm \\
4.15)\end{array}$ & \\
\hline LA maximum volume (ml) & $88 \pm 17$ & $67 \pm 21$ & $0.0001^{*}$ \\
\hline LA ejection fraction (\%) & $58 \pm 6$ & $56 \pm 9$ & 0.4 \\
\hline Extra-cellular volume (\%) & $\begin{array}{l}24.96 \pm \\
3.02\end{array}$ & $24.10 \pm 2.66$ & 0.3 \\
\hline
\end{tabular}

Values are mean \pm standard deviations or percentages. * signifies P?0.05. CMR-cardiac mag netic resonance imaging; n-numbers; LV-left ventricle; ml-milliliters; m-metre; $\triangle \mathrm{LVEF-change}$ in LV ejection fraction; gm-grams; s-seconds; RV-right ventricle; ?RVEF-change in RV ejection fraction; LA-left atrium. 